\title{
ÄSTHETISCHE ERFAHRUNG \\ UND QUASI-GEFÜHLE
}

\author{
Íngrid Vendrell Ferran
}

\section{Zusammenfassung}

Vor etwa einem Jahrhundert entwickelte sich im deutschsprachigen Raum im Rahmen einer allgemeinen Charakterisierung unserer ästhetischen Erfahrung von Kunst eine umfassende Debatte über die Natur und die Möglichkeit von Gefühlen über fiktionale Charaktere und Situationen. Die damalige Debatte weist große Ähnlichkeit zur heutigen analytischen Debatte über das Paradoxon der Fiktion auf. Trotz des unterschiedlichen jeweiligen historischen Kontextes findet sich in der analytischen Debatte und in der Philosophie zu Beginn des 20. Jahrhunderts fast der gleiche Lösungsansatz. Gefühle über Fiktionen seien Quasi-Gefühle, d.h. ein gefühlsartiges Phänomen mit einer Realität sui generis, dem im Unterschied zu den wirklichen Gefühlen kein Urteil über die Existenz des Objektes zugrunde liegt und dem die Verbindung zur Motivation fehlt. Ziel dieses Aufsatzes ist es, eine Untersuchung der These der Quasi-Gefühle Anfang des 20. Jahrhunderts zu unternehmen sowie die Analogien zur heutigen Debatte zu zeigen. Der Aufsatz baut sich um drei thematische Achsen herum auf. Zunächst wird eine historische Darstellung der These der Gefühle über Fiktionen bei Groos, Lipps, Lange, Geiger und Külpe unternommen. Diese bildet den Rahmen für die Debatte über die Quasi-Gefühle in der Grazer Schule. Anschließend wird die Aufmerksamkeit auf die Diskussion über QuasiGefühle bei Meinong, Witasek, Saxinger und Schwarz gerichtet. Zuletzt werden die Berührungspunkte zur heutigen analytischen Debatte im Ausgang von systematischen Fragen aufgezeigt.

\section{Vorbemerkung}

Trauer und Mitleid, Angst und Missgunst gegenüber fiktionalen Charakteren ergreifen uns ungeachtet der Tatsache, dass wir das Bewusstsein der 
Fiktionalität der Charaktere nicht verlieren. Dieses Phänomen ist einer der wertvollsten Aspekte unserer Erfahrung mit Kunst und scheint in der Natur unseres Daseins fest verwurzelt zu sein. So unumstritten aber diese Tatsache selbst ist, so sehr streiten Philosophen darüber, wie unsere emotionale Erfahrung von Fiktion zu verstehen ist. Sind jene Gefühle gegenüber fiktionalen Objekten derselben Natur wie die Gefühle über die Wirklichkeit?

Unter den verschiedenen Lösungsansätzen dieser Frage gibt es einen Ansatz, der fast wie eine Provokation klingt: die These der QuasiGefühle. Dieser Theorie zufolge sind Emotionen über Fiktionen keine echten Emotionen, sondern ein eigenartiges gefühlsähnliches Phänomen. Die These der Quasi-Gefühle wurde in der analytischen Tradition von Ryle, Kenny, Budd, Mulligan und ganz besonders von Walton entwickelt. Wenig bekannt ist aber, dass diese Theorie ihre Wurzeln in der Philosophie Humes hat und dass sie vor rund einhundert Jahren im deutschsprachigen Raum im Mittelpunkt der Diskussion über unsere ästhetische Erfahrung von Kunst stand. Ästhetiker wie Groos, Psychologen und Experimentalpsychologen wie Lipps und Külpe, Phänomenologen wie Geiger und ganz besonders die Autoren der Grazer Schule Meinong, Witasek, Saxinger und Schwarz haben Argumente für die These der Quasi-Gefühle entwickelt, die starke Ähnlichkeiten zu denen der heutigen Debatte aufweisen.

In diesem Aufsatz geht es mir darum, diese alte Lehre der Scheingefühle vom Anfang des 20. Jahrhunderts zu schildern und die Berührungspunkte zur heutigen Debatte freizulegen. Dafür werde ich zunächst die Theorien der Scheingefühle der damaligen Zeit darstellen. Dann werde ich ein besonderes Augenmerk auf die Debatte über die Natur der Quasigefühle in der Grazer Schule richten. ${ }^{1}$ Schließlich will ich die Verbindungen zur heutigen analytischen Debatte ans Licht bringen.

Ich werde auf Fragen psychologischer Natur achten und rein ontologische Fragen außer Acht lassen, obgleich es bei Meinong eine Theorie nicht existenter Objekte gibt, ausgehend von der man auch die Thematik der Quasi-Gefühle behandeln könnte (vgl. Raspa 2006; Barbero 2007). 


\section{Die Lehre von den Scheingefühlen}

Im Mittelpunkt des weiten Feldes der Ästhetik stand im 19. Jahrhundert die Frage nach dem Wesen der ästhetischen Erfahrung. ${ }^{2}$ Als Reaktion auf Kants Auffassung eines kontemplativen passiven Subjektes, betonten die damaligen Autoren das Gefühl und die aktive Teilnahme des Subjektes an der Suche nach dem ästhetischen Genuss. In diesem Rahmen entstand eine Vielzahl von Beiträgen über die emotionale Erfahrung mit Kunst, bei denen die Perspektive des Künstlers, des Konsumenten und gegebenenfalls des Schauspielers in den unterschiedlichen Ausübungen der Phantasie einbezogen wurde. Obgleich die Beschreibung der ästhetischen Erfahrung für alle Künste gelten sollte, erhielten in der damaligen Zeit die Mu$\operatorname{sik}^{3}$ und die „redenden Künste“ die größte Aufmerksamkeit. In dieser Debatte finden wir oft die Rede von „Scheingefühlen“ - der Terminus soll einen wichtigen Bestandteil unserer ästhetischen Erfahrung von Kunst bezeichnen. ${ }^{4}$

Wie sind „Scheingefühle“ zu verstehen? Für eine Minderheit von Autoren sind sie trotz all ihrer Besonderheiten den wirklichen Gefühlen gleich. Karl Gross ist ein Vertreter dieser Position, wonach die Gefühle, die den ästhetischen Schein begleiten, wirkliche, nicht bloß vorgestellte Gefühle sind (Gross 1902, S. 209). In derselben Linie vertritt Lipps die These, dass die Gefühle über Fiktionen eine besondere Unterklasse dessen sind, was er als Ernstgefühle bezeichnet. Es gibt Lipps zufolge eine Einstellungsänderung, wenn wir uns mit Fiktionen auseinandersetzen, die

2 Es gab dabei eine Wende von der Frage nach der Schönheit zur Frage nach der ästhetischen Erfahrung, vgl. Tatarkiewicz (2003); Huemer (2009), S. 267. Diese Frage steht zum Beispiel im Mittelpunkt bei Witasek (1904), Utitz (1911) und Külpe (1921), wenn auch bei einigen dieser Autoren wie Brentano und Witasek noch eine Spannung zwischen beiden Fragestellungen zu spüren ist.

3 In der Musikästhetik war die Diskussion über die Scheingefühle sehr lebendig. Eduard von Hartmann und Paul Moos vertraten die These, dass die Musik Ausdruck von Illusion und Täuschung ist. Theodor Vischer dagegen behauptete, dass sich bei der Musik ein reales Fühlen einstellt (vgl. Dorner 1975, S. 393400).

4 Geiger erwähnt hier Kirchmann, Th. Vischer, Hartmann, Moos, Witasek und Lange (vgl. Geiger 1914, S. 191). 
dazu führt, dass wir die Tatsachen der Fiktion ,hinnehmen“ (Lipps 1905, S. 492), ohne über das Dasein weitere Fragen zu stellen. Die Gefühle, die daraus entstehen, sind von einer anderen Qualität als die Gefühle über Wirkliches, aber sie sind für Lipps deswegen nicht weniger ernsthaft (Lipps 1905, S. 480).

Mehr Vertreter fand aber die entgegengesetzte Position, nach welcher die Erfahrung von Kunst uns in eine Welt des Scheins, der Illusion, des Spiels und des Als-Ob versetzt, so dass auch den darauf gerichteten Gefühlen dieser scheinhaften Charakter anhaftet. Wie - so fragen diese Autoren - wäre es sonst zu erklären, dass wir manchmal tragische Gefühle genießen können und genießen wollen? Es gibt eine Kluft zwischen Wirklichkeit und Schein, die sich in unser affektiven Erfahrung in beiden Bereichen widerspiegelt: Ästhetische Gefühle sind nicht wie die Gefühle der Wirklichkeit. In diesem Zusammenhang entwickelte sich ein breites Spektrum von Positionen über die Scheingefühle als Bestandteile der ästhetischen Erfahrung. Jeder der fraglichen Autoren versucht, die spezifische Natur der Scheingefühle gegenüber den auf Wirkliches gerichteten Gefühlen in unterschiedliche Weise zu erklären.

Eine radikale Ansicht entwickelte in dieser Hinsicht Konrad Lange in seiner Arbeit Das Wesen der Kunst (1901). Lange zufolge sind die Gefühle der dramatischen Poesie und Schauspielkunst, die Schriftsteller, Schauspieler, Leser oder Zuschauer empfinden, bloße „Gefühlsvorstellung, Illusionsgefühl oder Scheingefühl“ (Lange 1901, S. 104). Der Schriftsteller versetzt sich in die Gemütslage seiner erfundenen Figuren, der Schauspieler tut dasselbe, um die verschiedenen Charakteren so gut wie möglich zu verkörpern, und der Zuschauer oder Leser versetzt sich in die Phantasiewelt des Künstlers. Aus diesem Substrat der Phantasie heraus entsteht dann die Vorstellung und Illusion, ein Gefühl zu empfinden, ohne dass tatsächlich ein Gefühl vorliegt (Ebd., S. 97, 104). Es handelt sich hier nicht um eine Selbsttäuschung, denn Lange macht deutlich, dass wir das Bewusstsein der Fiktivität in den genannten Fällen nicht verlieren (Ebd., S. 100). Gefühlsvorstellungen unterscheiden sich von den Gefühlen der Wirklichkeit dadurch, dass sie ,etwas Gedämpftes, Gemäßigtes, gewissermaßen auf halbem Wege Steckengebliebenes" haben (Ebd., S. 100, 105). Damit wird nicht auf eine geschwächte Intensität abgezielt, sondern 
auf eine unterschiedliche Qualität des leiblichen Erlebens gegenüber den Gefühlen der Wirklichkeit und auch auf die Tatsache, dass Scheingefühle uns nicht zum Handeln veranlassen. Scheingefühle werden in dieser Auffassung auf bloße intellektuelle Akte des Vorstellens zurückgeführt und aus dem Reich der Gefühle verbannt.

Auch der Phänomenologe Moritz Geiger hat in seinem Vortrag „Das Problem der ästhetischen Scheingefühlen“ (1914) für die Lehre der Scheingefühle plädiert. Geiger zufolge fallen in diese Kategorie vier verschiedene Phänomene: 1) die Gefühle des Schauspielers auf der Bühne; 2) die Stimmungen, die durch unser Engagement mit Roman, Drama, Musik und Bild entstehen; 3) die Reaktionen auf Vorgänge auf der Bühne; 4) und die Sympathiegefühle für fiktionale Charaktere (Geiger 1914, S. 191192). Scheingefühle unterscheiden sich laut Geiger von wirklichen Gefühlen durch folgende Merkmale. Sie sind zeitlich begrenzt, sie sind situationsbedingt und sie zeigen eine ,weit geringere psychomotorische Wirksamkeit als die wirklichen Gefühle“ (Ebd., S. 192). Außerdem stört die Tatsache, dass sie manchmal sehr unlustvoll sein können, nicht den ästhetischen Genuss, sondern kann ihn sogar erhöhen (Ebd., S. 192). Die Scheingefühle sind demnach wie Nachzeichnungen echter Gefühlen, welche entstehen, wenn wir eine Spieleinstellung annehmen, bei der ich so tue, „als ob ich etwas erlebe, oder vorgebe, etwas zu erleben“ (Ebd., S. 193). In dieser Einstellung nehmen wir unsere Erlebnisse nicht ernst, wir sprechen ihnen kein volles Gewicht zu und entziehen den ästhetischen Gefühlen somit den Charakter des Realen. Die Spieleinstellung „nimmt dem Grauen, der Furcht, der Verzweiflung, Abscheu und Mitleid ihre Schwere und ihre Festigkeit und verleiht ihnen einen gewissen Scheincharakter" (Ebd., S. 194). ${ }^{5}$ Ähnlichen Beschreibungen lassen sich bei den Phänomenologen Voigtländer, Haas, Pfänder und Scheler finden, hier im Rahmen einer allgemeinen Theorie des unechten Psychischen (Voigtländer 1910; Haas 1910; Pfänder 1922; Scheler 1976).

Bei der Beschreibung des ,ästhetischen Zustands“ vertritt Oswald Külpe in Grundlagen der Ästhetik (1921) vehement die These der Scheingefühle. Nach einer Einstellungsänderung, bei der wir bereit zum ästheti- 
schen Verhalten sind und bei der wir uns auf einen bestimmten Gegenstand konzentrieren (Külpe 1921, S. 85), folgt eine Phase der Kontemplation, in der wir die Beschaffenheit des Objektes erleben (Ebd., S. 89). Erst dann sind die Gefühle möglich. Külpe erwähnt hier zum einem die „teilnehmenden Gefühle“, welche durch die Einfühlung in die Figuren der redenden Künsten entstehen: „Unser teilnehmendes Gefühl fürchtet für Egmont, bemitleidet Medea, verabscheut Richard III., trauert über den Untergang Gretchens“ (Ebd., S. 110). Hierbei handelt es sich um keine „vollständigen Gemütserregungen“, so Külpe, sondern um bloße Produkte einer „Haltung des Als-Ob“ (Ebd., S. 108). Zum anderen gibt es die Gefühle des ästhetischen Gefallens und Missfallens, welche laut Külpe echte Gefühle sind.

\section{Annahmen und Quasi-Gefühle bei Meinong}

\subsection{Spiel, Kunst und Annahme}

Das oben dargestellte Spektrum von Ansichten über die Scheingefühle bildet den Rahmen, in dem die Theorie der Quasi-Gefühle der Grazer Schule entwickelt wurde. ${ }^{6}$ Allerdings liegt der Kern von Meinongs Untersuchung des fraglichen Phänomens nicht in einer allgemeinen Beschreibung der ästhetischen Erfahrung und unserer affektiven Antworten auf dieselbe. Meinong ist vielmehr an der Natur einer besonderen Art intellektueller Vorgänge interessiert, die unsere Erfahrung mit Spiel und Kunst leitet: den Annahmen.

In Über Annahmen (1902) wendet Meinong sich zunächst den Kinderspielen zu und überträgt die hier gefundenen Muster auf die Spiele der Erwachsenen und die Kunst, die er als eine Form des Spiels betrachtet. Meinong fragt nach den intellektuellen Vorgängen bei dem Schriftsteller, dem Schauspieler und dem Leser, die sich in die fiktionalen Figuren „hin-

5 Diese Thesen werden Geiger und Ortega y Gasset später weiterentwickeln (vgl. Geiger 1974; Ortega y Gasset 2005).

6 Für eine detaillierte Charakterisierung der Quasi-Gefühle in der Grazer Schule vgl. Smith (1994), S. 131-134. 
einversetzen“. Seine These lautet, ,dass der Spielende an sich und anderen Eigenschaften, Situationen u. dgl. „,ingiere“, um dann häufig, solange das Spiel währt, zu tun, als ob er an die Fiktion glaubte, obwohl ihm solches völlig ferne liegt" (Meinong 1977, S. 112). Hier handelt es sich nicht um eine Täuschung hinsichtlich Fiktion und Realität, denn sogar kleine Kinder unterscheiden deutlich zwischen Spiel und Ernst (Meinong 1977, S. 111; Harris 2000, S. 60), sondern um eine bestimmte intellektuelle Einstellung, welche Meinong als „Annahme“ bezeichnet. Diese These (Meinong 1977, S. 115) hat wichtige Implikationen für Meinongs Auffassung der Scheingefühle.

Den Begriff des Scheingefühls entwickelt Meinong im Zusammenhang mit dem Begriff der Ernstgefühle. Meinong spricht aber nicht von Scheingefühlen, sondern von Phantasie- und Quasi-Gefühlen. In Meinongs Bild des Psychischen gibt es vier Hauptarten psychischer Akte: Vorstellungen, Urteile, Gefühle und Begehrungen. Meinong zufolge kann jede dieser vier Hauptklassen in zwei Formen auftreten: als ernsthaft oder als schattenhaft (Meinong 1923, S. 128-129). Letzteres geschieht, wenn die psychischen Akte durch die Leistung der Phantasie „modifiziert“ werden. Daraus entstehen vier Gegenstücke der vorher erwähnten psychischen Akte: Phantasievorstellungen und Erinnerungen, Phantasieurteile oder Annahmen, Phantasiegefühle und Phantasiebegehrungen (Meinong 1923 und 1977).

Die schattenhaften Gegenstücke entstehen ausgehend von der Ausübung der kontrafaktischen Phantasie. Bei all diesen psychischen Akten fehlt das Moment des Überzeugtseins von der Existenz des Objekts (Smith 1994, S. 128; Meinong 1977). Phantasievorstellungen und Erinnerungen sind psychische Akte, denen anders als ihren ernsthaften Gegenstücken die Überzeugung von der Existenz ihres Objektes fehlt. Dasselbe gilt für die Annahmen und die Phantasiegefühle. Annahmen versteht Meinong als urteilsähnliche Phänomene, die mehr als ein bloßes Vorstellen sind, da hier anspruchsvollere kognitive Prozesse verlangt werden, und die gleichzeitig weniger sind als Urteile, da sie kein Existenzurteil implizieren. Sie sind daher ein psychischer Akt sui generis, dem eine „Zwischenstellung" zwischen Vorstellungen und Urteilen zukommt (Meinong 1977, S. 112, im selben Sinne S. 111 und 309). Auch die Phantasiegefühle 
sind eine Klasse psychischer Akte sui generis, die eine hybride Natur zwischen Vorstellung und Gefühl beanspruchen. Phantasiegefühle sind nicht Vorstellungen, da sie wie die Klasse der Gefühle der Gegensätzlichkeit von Lust und Unlust unterliegen (Meinong 1977, S. 312). Diese Gegensätzlichkeit ist weder in der Klasse der Vorstellungen noch der Urteile zu finden, da erstere keine Gegensätzlichkeit aufweisen und letztere zwischen Affirmation und Negation schwanken. Allerdings weigert sich Meinong, Phantasiegefühle als echte Gefühle zu verstehen. Worin liegt nun der Unterschied zwischen beiden Gefühlsarten?

\subsection{Gefühle, Phantasiegefühle und Quasi-Gefühle}

Gefühle werden laut Meinong durch einen leiblichen und einen kognitiven Aspekt gekennzeichnet. Als leibliches Moment versteht Meinong hauptsächlich die Tatsache, dass Gefühle immer entweder lustvolle oder unlustvolle Erfahrungen sind (Meinong 1923, S. 132). Meinong verweist auch auf eine spezifische Qualität, in der die Gefühle leiblich „,verspürt“ werden, ohne diesen Punkt allerdings weiterzuentwickeln. Das kognitive Moment der Gefühle besteht darin, dass Gefühle notwendigerweise in intellektuellen Akten wie Vorstellungen und Urteilen gründen und dass sie mit Werten verbunden sind (vgl. Vendrell Ferran 2009).

Phantasiegefühle schwanken zwischen Lust und Unlust und sie haben denselben Namen wie ihre ernsthaften Gegenstücke (Meinong 1977, S. 313). Beide Gefühlsarten unterschieden sich aber zum einem leiblich in der Tatsache, dass Phantasiegefühle mit einer anderen Qualität erlebt werden. Zum anderen besteht auch auf der kognitiven Ebene ein Unterschied: Während Ernstgefühle ernsthafte Akte wie Vorstellungen und Urteile zur Voraussetzung haben, gründen die Phantasiegefühle in schattenhaften Phantasievorstellungen (d.h. Phantasiewahrnehmungen und Erinnerungen) und Annahmen. Die Angst, die ich bei der Phantasiewahrnehmung eines Gespenstes empfinde und die Angst, die an die Erinnerung an einen Hagelsturm anknüpft, sind Beispiele für Phantasiegefühle, die in Phantasievorstellungen gründen. Ein Phantasiegefühl ist auch die Trauer, die ich bei der Annahme des Unglücks Anna Kareninas empfinde. Für 
diesen besonderen Fall von Phantasiegefühlen, die in Annahmen gründen, prägt Meinong den Terminus „Quasi-Gefühl“. Über die Quasi-Gefühle behauptet er: „In der Tat, jene ,Furcht' und jenes ,Mitleid“, oder was sonst die Tragödie zu ,erwecken' die Aufgabe haben mag, was sind sie eigentlich? Eine Furcht, bei der man sich im Grunde doch gar nicht fürchtet, ein Mitleid, das näher besehen eigentlich doch gar kein Weh verspüren lässt, sind das noch ,Gefühle“, wie man sie in der Psychologie zunächst zu behandeln pflegt?“" (Meinong 1977, S. 310). Meinongs Antwort ist so deutlich wie radikal: Phantasiegefühle und ihre Unterklasse der Quasigefühle sind keine Gefühle. Wären sie real, so die Mutmaßung, dann würden wir uns keine Tragödie anschauen und keinen Roman mit einem tragischen Ende lesen wollen.

Meinong spricht den Quasi-Gefühlen den Ernstcharakter aus drei Gründen ab. Erstens gründen sie in Annahmen - also nicht in ernsthaften psychischen Akten. Wenn wir uns mit Fiktionen engagieren, haben wir Annahmen über die Welt der Fiktion, ohne uns hinsichtlich der Existenz des Fiktionalen weitere Fragen zu stellen. Wir nehmen einfach an, was der Schriftsteller oder Künstler uns vermittelt. Diese Annahmen sind die kognitiven Grundlagen, auf denen die Quasi-Gefühle fußen. Es gibt daher einen Unterschied in dem kognitiven Moment beider Gefühlsarten: Während Ernstgefühle in ernsthaften psychischen Akten wie Vorstellungen und Urteile gründen, basieren die Quasigefühle auf Annahmen. ${ }^{7}$ In dieser These steckt ein sehr normativer Begriff der Gefühle, bei dem der Akzent besonders auf die Urteile als kognitive Basis der Emotionen gelegt wird. Urteile sind die wesentliche kognitive Basis der Gefühle, und da die Quasi-Gefühle in Annahmen gründen, werden sie aus dem Reich des echten Fühlens verbannt.

Ein weiteres Merkmal, das Quasi-Gefühle von den echten Gefühlen unterscheidet, betrifft ihre „Leiblichkeit“. Meinong behauptet, dass Qua-

7 Diese These ist - wie Barry Smith gezeigt hat - problematisch. Zum einen können wir echte Gefühle haben, bei denen das Moment der Konviktion hinsichtlich der Existenz des Objekts nicht fehlt, obwohl das Objekt nicht existiert (ein Beispiel sind die kindlichen Gefühle für den Weihnachtsmann). Zum anderen können wir Phantasiegefühle über existente Objekte haben, an die wir nicht glauben (vgl. Smith 1994, S. 131). 
si-Gefühle mit einer anderen Qualität als die Ernstgefühle erlebt werden. Sie sind demnach „schattenhaft“, sie sind keine Gefühle im „eigentlichen Sinne“ und sie werden als ,uneigentlich verspürt“ (Meinong 1977, S. 313) - all dies, weil sie in nicht ernsthaften psychischen Akten gründen. Diese Ansicht wurde schon von Meinongs Zeitgenossen wie Lange, Geiger und Külpe vertreten, wenn sie die Scheingefühle als ,gedämpftes, gemäßigtes Steckengeblieben“, als „scheinhaftig“ oder als „unvollständiges" Phänomen beschreiben. Innerhalb der Grazer Schule selbst aber gab es diesbezüglich weniger Einklang. Saxinger und Schwarz werden die Meinong'sche These argumentativ und experimentell zu verteidigen versuchen, Witasek dagegen wird für die These plädieren, dass beide Gefühlsarten physiologisch und phänomenologisch nicht zu unterscheiden sind und daher der ,emotionale Faktor“ - wie er es bezeichnet - der gleiche ist.

Der Ursprung von Meinongs These ist in der Philosophie Humes zu finden. ${ }^{8}$ In A Treatise of Human Nature vertritt Hume eine kognitivistische Auffassung der Gefühle insofern, als die „passions“ immer Urteile zur Voraussetzung haben (Hume 2008, S. 82, auch 274). Im Rahmen dieser kognitivistischen Auffassung der Gefühle entsteht die Frage danach, wie die Gefühle gegenüber demjenigen, woran wir nicht glauben, zu erklären sind. Laut Hume ist die Welt der Dichtung bloße Erscheinung von Überzeugungen, bloße Scheinwelt und sogar Lüge; die Möglichkeit des Urteilens sei hier von vorneherein ausgeschlossen (Ebd., S. 83 und 85). Damit etabliert er einen Unterschied zwischen den Gefühlen, die sich auf die Wirklichkeit beziehen, und denen, die sich auf Fiktionen beziehen, da letzteren das Moment der Überzeugung an der Existenz des Objektes fehlen soll. Dieser Unterschied beider Gefühlsarten wird wie folgend zusammengefasst: „A passion, which is disagreeable in real life, may afford the highest entertainment in a tragedy, or epic poem. In the latter case it lies not with that weight upon us: It feels less firm and solid: And has no other than the agreeable effect of exciting the spirits, and rouzing the at-

8 Der Einfluss Humes ist bei Brentano und seinen Schülern, besonders Meinong und Husserl, deutlich zu spüren. Die Philosophie Humes stellte eine Alternative zum damals herrschenden Kantischen Modell der Psyche dar, welches Brentano und seine Schüler so entschieden bekämpften. Vgl. Kühn (2006), S. 98-99. 
tention. The difference in the passions is a clear proof of a like difference in those ideas, from which the passions are derived" (Ebd., S. 85). Dieser Ansicht zufolge werden die Quasi-Gefühle mit einer gewissen Distanz erlebt, so dass es dann auch möglich ist, unlustvolle Gefühle zu genießen. Die Quasi-Gefühle haben außerdem weniger Gewicht und sie sind - so Hume - weniger fest und solide als die Gefühle der Wirklichkeit. Diese Distanz erklärt für den Philosophen, warum wir manchmal sehr unlustvolle Gefühle in der Fiktion erleben wollen. ${ }^{9}$ Diese ganze Terminologie wird von Meinong übernommen, sie ist auch in der Phänomenologie Voigtländers, Haas' und Pfänders zu finden und sie bedingt sogar die analytischen Beschreibungen der Quasi-Gefühle bis in unsere Tage hinein, wie die Beschreibungen von Ryle, Kenny, Budd, Walton, Mulligan und Pugmire bezeugen (Ryle 1963, S. 103; Kenny 1963, S. 49; Budd 1985, S. 128; Walton 1978, S. 6, und 1990, S. 196; Mulligan 1992; Pugmire 1994, S. 105, und 2005, S. 36).

Ein letztes Kennzeichen der Quasi-Gefühle gegenüber echten Gefühlen betrifft ihre Verbindung zur Motivation. Während echte Gefühle motivationale Kraft haben, können laut Meinong Quasi-Gefühle keine Handlungen veranlassen. Meinong behauptet, dass Quasi-Gefühle nicht mit echten Wünschen zusammen auftreten: „der urteilsfähige Romanleser hat für die Personen des Romans ganz ebenso Wünsche, als er Gefühle für sie hat: und diese Wünsche sind streng genommen genau so wenig eigentliche Wünsche, als jene Gefühle eigentliche Gefühle sind“ (Meinong 1977, S. 315). In diesem Zusammenhang spricht er von „Phantasiebegehrungen“" (Meinong 1977, S. 314).

Meinong zufolge sind Quasi-Gefühle ein gefühlsartiges Phänomen mit einer eigenen Realität sui generis, das Gefühlen ähnelt, jedoch kein echtes Gefühl darstellt. Sie verdienen den Namen „Gefühl“, weil sie eine leibliche Erfahrung sind, welche als lustvoll oder unlustvoll charakterisiert werden kann. Aber sie sind keine vollwertigen Gefühle, weil sie auf Annahmen gründen, weil sie mit weniger Gewicht erlebt werden und weil

9 In „Of Tragedy“ behauptet Hume, dass in den Fällen, in welchen das Lustgefühl gegenüber der künstlerischen Darstellung größer ist als das Unlustgefühl über den Inhalt der Darstellung, aus Unlustgefühlen Lustgefühle werden (Hume 2004). Vgl. für eine Kritik Currie \& Ravenscroft (2002), S. 199. 
ihnen die Verbindung zur Motivation fehlt. Jede dieser Thesen wurde von anderen Autoren der Grazer Schule entwickelt, dabei bildete sich eine lebhafte Diskussion über Witaseks alternative Auffassung der QuasiGefühle und die Beiträge von Saxinger und Schwarz, die Meinongs Auffassung gegen Witasek verteidigten.

\section{Quasi-Gefühle und ästhetische Erfahrung bei Witasek}

\subsection{Witaseks alternative Auffassung der Quasi- Gefühle}

Während Meinong zu seiner These der Quasi-Gefühle von der Untersuchung der intellektuellen Vorgänge bei Annahmen aus gelangt, entwickelt Witasek seine alternative Auffassung im Rahmen einer umfassenden Analyse der ästhetischen Erfahrung. In Grundzüge der allgemeinen Ästhetik (1904) untersucht Witasek, wie jeder der unterschiedlichen ästhetischen Gegenstände uns in der Erfahrung gegeben wird. Der Fokus von Witaseks Ästhetik pendelt dabei zwischen der Frage nach der Schönheit und der psychologischen Analyse der verschiedenen psychischen Zustände des Subjekts, bei denen der ästhetische Genuss eine Rolle spielt. ${ }^{10}$

Witasek zufolge gibt es fünf verschiedene ästhetische Gegenstände, die als Träger von „Schönheit" fungieren: einfache Empfindungsgegenstände, Gestalten, normgemäße Gegenstände, das Ausdruck- und Stimmungsvolle und das Objektive (Ereignisse, Zustände usw.). Jeder dieser Typen ist mit einem bestimmten ästhetischen Zustand oder ästhetischen Verhalten des Subjekts verbunden (Witasek 1904, S. 19). Das Thema der

10 Der Einfluss Brentanos auf Witaseks Ästhetik ist ein dreifacher. Witasek zielt erstens darauf ab, die Ästhetik in der psychologischen Untersuchung des Subjekts bei der ästhetischen Erfahrung zu gründen, genauso wie die Logik und die Ethik in der Psychologie gründen müssen, ohne dass es zu einem Abfall in den Psychologismus kommt. Trotz dieser Analyse der ästhetischen Erfahrung verstehen beide Autoren zweitens die Ästhetik als Wissenschaft des Schönen und beide Autoren verstehen drittens die ästhetische Erfahrung hauptsächlich als Genuss vgl. Huemer 2009, S. 273, 283, 285). 
Quasi-Gefühle tritt nun bei der Beschreibung der ausdrucks- und stimmungsvollen Objekte wie Skulpturen, Dramen und Romanen auf. Da Witasek die ästhetische Erfahrung stark mit dem Konzept des ästhetischen Genusses verbindet, entsteht für ihn die Frage, wie es möglich ist, dass wir bei dem Genuss von Kunst manchmal die ästhetische Erfahrung von Leid oder Trauer haben wollen. Um dieses Rätsel zu lösen, unterscheidet Witasek zwischen dem echten Gefühl des ästhetischen Genusses einerseits und den Gefühlen angesichts der Ausdrucks- und Gestaltobjekte andererseits, welche er als "Quasi-Gefühle“ bezeichnet (Witasek 1904, S. 110). Witasek präsentiert eine ausgearbeitete Untersuchung dieser QuasiGefühle, die Meinongs Analyse zwar ähnelt, aber auch einige entscheidende Unterschiede aufweist.

Quasi-Gefühle sind laut Witasek, der in diesem Punkt Meinongs Bild des Psychischen teilt, Spiegelbilder der echten Gefühle in dem Feld der Phantasie. Diese Spiegelbilder sollen eine Stellvertreter-Funktion haben (Witasek 1904, S. 110 und 119). Das Hauptkennzeichen der QuasiGefühle ist demnach, dass sie nicht auf Urteilen, sondern auf Annahmen gründen. Witasek schreibt: ,[...], dass alle Phantasiegefühle Annahmen zur Voraussetzung haben, niemals ein Urteil, und daß umgekehrt Annahmen, sofern sie als Gefühlsvoraussetzung fungieren, immer zu Phantasiegefühlen gehören“ (Ebd., S. 118, im selben Sinne S. 116). ${ }^{11}$ Alle Gefühle, die in Annahmen gründen, sind daher als Phantasiegefühle zu klassifizieren.

Diese These ist der Ausgangspunkt einer starken Kontroverse mit Meinong, denn laut Witasek ist jedes Phantasiegefühl ein Annahmegefühl. Laut Meinong können Phantasiegefühle hingegen sowohl auf Phantasievorstellungen als auch auf Annahmen gründen. Witaseks These impliziert, dass Quasi-Gefühle nicht per se eine Klasse psychischer Phänomene sind, da sie bloß Gefühle sind, die auf Annahmen gründen.

Meinong kritisiert Witaseks Ansicht auf zweifache Art. Zum einen zeigt er, dass nicht alle Phantasiegefühle Annahmen zur Voraussetzung

11 Witasek selbst aber scheint auch in seinem Text die Möglichkeit zu erkennen, dass es Phantasiegefühle gibt, die auf Phantasievorstellungen statt auf Annahmen gründen. Etwa wenn man sich ein schönes Ornament oder ein Tonstück anschaulich vorstellt und dann Lust oder Unlust empfindet. 
haben. Es gibt Phantasiegefühle, die in Erinnerungen gründen, wie das Gefühl, welches entsteht, wenn wir uns an einen Zahnschmerz erinnern, oder wenn wir uns an eine Trauer erinnern und dann noch einmal all die Urteile fällen, die Voraussetzung dieser Trauer waren. Der Bereich der Annahmegefühle ist demnach viel enger als der Bereich der Phantasiegefühle (Meinong 1977, S. 317). Zum anderen argumentiert Meinong, dass nicht alle Gefühle, die Annahmen als Voraussetzung haben, Phantasiegefühle sind. Es gibt laut Meinong in der Ästhetik die Möglichkeit von Ernstgefühlen, die auf Annahmen gründen (Meinong 1977, S. 319). Saxinger und Schwarz werden weitere Argumente zugunsten der Meinong'schen Ansicht entwickeln.

Ein weiterer wichtiger Aspekt, der Witaseks Ansicht von Meinongs Ansicht stark unterscheidet, betrifft den „emotionalen Faktor“ der Gefühlen. Witasek behauptet, dass beide Gefühlsarten - Quasi-Gefühle und vollwertige Gefühle - phänomenologisch und physiologisch gleichartige Phänomene sind (Witasek 1904, S. 114, 116). Folgendes Beispiel soll dies laut Witasek untermauern: Wenn wir in einer bestimmten psychischen Lage sind, etwa Unlust, sind wir nicht imstande, ein lustvolles Phantasiegefühl vorzustellen. Schaffen wir es doch, ist die ursprüngliche Unlust bereits gebrochen. Auch ist es für uns schwierig, uns wenn wir fröhlich sind in die Gemütslage des Elenden zu versetzen ,und auch da tut es der Ernst-Lust Abbruch, wenn es trotzdem geschieht“ (Ebd., S. 119). Dies geschieht, weil der „emotionale Faktor“, den Witasek hauptsächlich als Lust und Unlust versteht, von Ernst- und Phantasiegefühlen sehr ähnlich ist. Wenn sie wesentlich anders wären, gäbe es kein Problem, beide Gefühlsarten gleichzeitig zu empfinden. Meinong dagegen behauptet, dass QuasiGefühle eine andere Qualität des Erlebens zeigen, insofern sie irgendwie schal und hohl erscheinen. Während Witasek darüber hinaus den „emotionalen Faktor" als Lust und Unlust versteht, scheint Meinong damit eine bestimmte Form des Erlebens zu bezeichnen, die nicht nur die hedonistische Valenz betrifft.

Weniger problematisch sind die anderen Merkmale, die Witasek für die Quasi-Gefühle angibt. Hier finden wir erneut die These, dass QuasiGefühle mit einer gewissen Distanz erlebt werden und ,weder freuen noch schmerzen“ (Witasek 1904, S. 115). Anderenfalls würde sich kein 
Mensch eine Tragödie anschauen wollen, wenn nämlich der Schreck, die Sorge, das Mitleid und die Furcht und alle anderen oft intensiven Unlustgefühle echt wären. Quasi-Gefühlen fehlt auch nach Witasek eine Verbindung zur Motivation, da sie bloß mit Phantasiebegehrungen zusammen auftreten. Schließlich behauptet Witasek wie die anderen Autoren der Grazer Schule, dass wir Quasi-Gefühle in unserer Gewalt haben: „,wir lassen sie uns gefallen, wenn es uns Freude macht oder zu etwas dienlich ist, wenn sie uns aber unbequem werden, löschen wir sie kraft der souveränen Macht, die wir über die Annahmen haben, einfach aus“" (Ebd., S. 116, 117). Dabei erkennt Witasek durchaus die Möglichkeit an, dass wir die Gefühle bei einer Theateraufführung nicht mehr im Griff haben und dann gegebenenfalls das Theater verlassen müssen.

\subsection{Gefühlssuggestionen, Gefühlsgestalten und psychische Resonanz}

Das interessante an Witaseks Beitrag über die Quasi-Gefühle in Grundzüge der allgemeinen Ästhetik liegt nicht nur an der oben erwähnten Diskussion mit Meinong, sondern vor allem an der detaillierten psychologischen Analyse dieses Phänomens, die in einigen Zügen sehr innovativ ist. Witasek geht von einer Analyse der Quasi-Gefühle in der Musik aus und überträgt die Ergebnisse dann auf das Schauspiel, die Bildhauerei, die Architektur und die „Dichtkunst“".

Bei der Bildung von Phantasiegefühlen spielt in Witaseks Modell der Begriff der „Gefühlssuggestion“ eine wichtige Rolle. Gefühle, die bei der Musik und Dichtkunst entstehen, sind laut Witasek Quasi-Gefühle, weil ihnen die nötigen Voraussetzungen für vollwertige Gefühle fehlen. Wir empfinden Trauer oder Mitleid aufgrund einer bloßen Wahrnehmung von Tönen, ohne dass es ein entsprechendes Urteil etwa über einen Verlust, ein Unglück usw. gegeben hat. Diese Gefühlssuggestionen sind laut Witasek dem Willen unterworfen: „Auch ohne irgend welche äußere Hilfe ist man schon durch den bloßen Willen dazu imstande, Phantasiegefühle verschiedener Art, z. B. Zorn, Ärger, Erfurcht im eigenem Inneren mehr oder minder lebhaft, im ganzen freilich nur in geringer Intensität anklingen zu 
lassen“ (Witasek 1904, S. 136). Wie dies geschehen soll, erklärt Witasek mithilfe zweier weiterer Begriffe: dem der „psychischen Resonanz“ und dem der ,Gefühlsgestalt“.

Der Mechanismus der „psychischen Resonanz“ besteht darin, ein bestimmtes Gefühl und die entsprechenden psychischen Begleiterscheinungen in Erinnerung zu rufen, so dass dann das Gefühl auch entsteht (Witasek 1904, S. 136). ${ }^{12}$ Mit dem Terminus „Gefühlsgestalt“ verweist Witasek auf die Tatsache, dass die konkreten psychischen emotionalen Erlebnisse eine bestimmte Form und einen bestimmten zeitlichen Ablauf haben. Jedes unserer Gefühle wird demnach durch eine bestimmte Gefühlsgestalt bestimmt: „Es ist eine andere Gestalt, in der sich die Unlust abspielt im Zorn, eine andere in der Reue; dort ist ein beständiges aber regelloses Anund Abschwellen, eine gewaltige Bewegung, hier ein mehr ruhiger, gleichsam schneidender und bohrender Druck“ (Ebd., S. 140). Die Vielfalt der Gefühlsgestalten ist laut Witasek sehr groß. Ihm zufolge bedient sich jede Kunst verschiedener Materialien, um das Phantasieleben anzuregen und die Gefühlsgestalten in Musiktönen, Figuren oder Worten zu wecken, so dass dann die entsprechende psychische Resonanz zustande kommt. Im Fall der Musik gibt es eine Ähnlichkeit zwischen Tongestalten und Gefühlen, die der Musiker benutzt, um in uns Gefühle zu erwecken. In der Dichtkunst liefert uns die Sprache die Urteile, Annahmen und Beschreibungen als Basis für die Hervorrufung eines Gefühls. Außerdem werden in der Literatur und im Theater die Gefühle und Stimmungen der Personen und Situationen selbst geschildert, so dass der Leser oder Zuschauer sie nacherleben kann. Die Reproduktion der psychischen Gefühlsresonanz kann auch durch die Sprachmelodie und den Rhythmus angeregt werden und natürlich ist hier auch nicht zu vergessen, dass der In-

12 Auch Ortega y Gasset hat auf die Bedeutung dieses Mechanismus' im Fall der Musik hingewiesen und auf seine Rolle bei der Schaffung eines Musikstücks mit einer bestimmten Gefühlsgestalt Er erklärt im Kontext der romantischen Musik, dass Beethoven von einer wirklichen Situation ausgehe, dann diese Situation Gefühle in sich auslösen lasse, den entsprechenden Gefühlswallungen folge und schließlich versuche, diese in Musik zu übersetzen. Diese Behauptung ist in der Richtung zu interpretieren, dass er den Emotionen bestimmte Qualitäten zuschreibt, mit denen sie am Leib gefühlt werden. Vgl. Ortega y Gasset (1954), S. 349. 
halt, das Erzählte und Dargestellte, eine wichtige Rolle spielt. Alle diese Mechanismen werden in der heutigen Debatte der Philosophie der QuasiGefühle kaum beachtet, obgleich sie Teil unseres psychologischen Engagements mit Fiktionen sind (Ausnahmen hier sind: Feagin 2004; Gholamain \& Oatley 1997, S. 273).

\section{3 Ästhetischer Genuss in der Dichtkunst}

Neben den Begriffen der Schönheit und der ästhetischen Erfahrung spielt der Begriff des ästhetischen Genusses in Witaseks Ästhetik eine wichtige Rolle. Der Autor veranschaulicht ihn anhand der Dichtkunst und nennt dabei drei Faktoren, die für den ästhetischen Genuss wichtig sein sollen.

Voraussetzung für den ästhetischen Genuss sind für Witasek erstens die Einfühlung und die Anteilsgefühle. Unter Einfühlung versteht er ein „Vorstellen von Gefühlen“ anderer (Ebd., S. 120) und nennt als Beispiel das Mitleid, das wir mit Fausts Gretchen empfinden. Die Anteilsgefühle dagegen sind die Reaktionen des Subjektes auf die Erzählung; als Beispiele dienen etwa die Zuversicht oder die Verzweiflung, die wir empfinden, wenn wir das Drama lesen (Ebd., S. 149). In beiden Fällen handelt es sich um Phantasiegefühle, welche als Voraussetzung des echten ästhetischen Genusses fungieren (Ebd., S. 150).

Der ästhetische Genuss setzt zweitens voraus, dass wir das Bewusstsein besitzen, dass es sich um Phantasiegefühle handelt. Witasek spricht hier von einer gewissen Distanz zu den eigenen Phantasiegefühlen und von einer unernsten Einstellung zur Fiktion. Wer mitten bei der Lektüre oder bei der Aufführung eines Theaterstücks die Aufmerksamkeit auf sich selbst lenkt, für den gilt laut Witasek: „Er wird sich gleichsam dabei ertappen, wie er bei der Betrachtung der vorgeführten Dinge und Ereignisse doch auch den Vorgängen in der eigenen Seele lauscht, wie er die eigene emotionale Reaktion [...] beobachtet und manchmal, wenn sie in höchster Intensität sich regt, ganz ausdrücklich und mit Bedacht die Aufmerksamkeit auf sie lenkt und so des höchsten ästhetischen Genusses teilhaftig wird“ (Ebd., S. 153-154). Dieser Aspekt wird auch von Geiger und im Anschluss an ihn von Ortega y Gasset untersucht werden. Geiger wird 
von Innen- und Aussenkonzentration sprechen. Erstere liegt vor, wenn wir keine Distanz haben und uns vollkommen den Gefühlen des Dramas oder Romans hingeben; die Außenkonzentration erlaubt uns dagegen, auf die Qualitäten des Kunstwerks zu achten, ohne von den Gefühlen hingerissen zu werden (Geiger 1974, S. 63). Schließlich spielt drittens auch die Spannung eine große Rolle als Genussfaktor im Fall von Roman und Drama.

Witaseks Auffassung der ästhetischen Erfahrung offenbart sich hinsichtlich der Bewertung der Gefühle als radikal: Alle Gefühle, die wir im Laufe unseres Engagements mit Kunst haben, sind als unecht zu bezeichnen - mit einer Ausnahme: der des ästhetischen Genusses. Meinong stellte als Reaktion auf Witasek die Frage: „Wo bleibt da noch Raum für ästhetische Ernstgefühle?“ (Meinong 1977, S. 319). Die einzige Antwort lautet: In der ästhetischen Erfahrung des Genusses.

\section{Einfluss der Phantasie auf die Gefühle: Robert Saxinger und Ernst Schwarz}

\subsection{Phantasiegefühle als Gefühlsvorstellungen}

Robert Saxinger und Ernst Schwarz haben die Thematik der QuasiGefühle in einer Reihe von Artikeln behandelt. Saxinger in „Dispositionspsychologisches über Gefühlskomplexionen“ (1902), „Über den Einfluss der Gefühle auf die Vorstellungsbewegung“ (1902), „Über die Natur der Phantasiegefühle und Phantasiebegehrungen“ (1904), „Beiträge zur Lehre von der emotionalen Phantasie“ (1906) und „Gefühlssuggestion und Phantasiegefühl“ (1908) und Schwarz in „Über Phantasiegefühle“ (1906) - beide versuchen in den genannten Schriften, Meinongs Auffassung der Phantasiegefühle gegenüber Witaseks Alternative argumentativ und experimentell zu verteidigen. Ihnen geht es darum, die Phantasiegefühle als eine Klasse eigenständiger psychischer Grundphänomene zu charakterisieren (Saxinger 1904, S. 581). Ein Blick auf die Untersuchungen zeigt dabei, dass beide Autoren ihre Arbeit auf folgende Fragen konzentrieren: Sind Phantasiegefühle bloße Gefühlsvorstellungen, also lediglich vorgestellte Gefühle? Folgen beide Gefühlsarten denselben Geset- 
zen? Kann ein Gefühl durch Suggestion erzeugt werden? Gibt es Phantasiegefühle, die in Phantasievorstellungen gründen? Und: Was für eine Klasse psychische Phänomene sind?

Im Hintergrund des Forschungsprogramms beider Autoren stehen Humes Anmerkungen zum Einfluss der Imagination auf die Gefühle in $A$ Treatise of Human Nature. ${ }^{13}$ In diesem Text untersucht Hume die Erinnerung an vergangene Gefühlen und deren Einfluss auf zukünftige Gefühle sowie die Kraft der Gefühle, die fest in das psychische Leben eingebettet sind, gegenüber der von denjenigen Gefühlen, die bloß durch die Ausübung der Imagination entstehen; zuletzt befasst sich Hume mit der Fähigkeit von Sprache, Gefühle hervorzubringen (Hume 2008, S. 273). Diese verschiedenen Aspekte spiegeln sich in den Untersuchungen von Saxinger und Schwarz wider.

Wenden wir uns zunächst der ersten Frage zu, ob Phantasiegefühle Gefühlsvorstellungen im Sinne Konrad Langes sind. Laut Saxinger sind Phantasiegefühle weder vorgestelle Gefühle noch angenommene Gefühle. Vorstellen und Annehmen sind intellektuelle Vorgänge ,während die fraglichen Phänomene nach dem Zeugnis der inneren Wahrnehmung unzweifelhaft in das Gebiet der emotionalen Erscheinungen einzureihen sind.“ Außerdem sind Vorstellungen und Annahmen laut Saxinger „farblose psychische Erlebnisse“ (Saxinger 1904, S. 581) im Vergleich zu den Gefühlen. In diesem Punkt folgt er Meinongs Thesen.

Schwarz vertritt eine ähnliche Ansicht und nennt zwei weitere Argumente gegen die These von Phantasiegefühlen als bloßen Vorstellungen von Gefühlen. Schwarz zeigt zum einem, dass die Phantasiegefühle zwischen den Gegenpolen von Lust und Unlust schwanken. Dieses Merkmal ist typisch für die Klasse der Gefühle und lässt sich bei den Vorstellungen nicht finden (Schwarz 1906, S. 496).

Zum anderen untersucht Schwarz die Frage, ob Phantasiegefühle bloße Reproduktionen von Ernstgefühlen sind, so wie Phantasievorstellungen Reproduktionen von Wahrnehmungen und Erinnerungen sind. Er kommt zu dem Schluss, dass der Begriff der Reproduktion sich auf den Bereich der Gefühle nicht anwenden lässt. Phantasiegefühle sind laut 
Schwarz keine bloßen Vorstellungen von Gefühlen, da Phantasiegefühle auch dann erlebt werden können, wenn die entsprechenden Ernstgefühle nicht erlebt worden sind. Dies geschieht etwa dann, wenn wir ausgehend von einer Phantasiewahrnehmung, Erinnerung oder Annahme ein Phantasiegefühl erleben, dessen vollwertiges Äquivalent wir nicht kennen. Wenn auch diese Phantasiegefühle keine psychischen Dispositionen begründen können sollen (Schwarz 1906, S. 102), so verweisen sie doch auf die Möglichkeit, dank der Kraft der Phantasie unser Gefühlsleben zu bereichern. Damit wird der Wert der Kunst für die Erweiterung unseres emotionalen und kognitiven Horizonts hervorgehoben. In diesen Ausführungen steckt auch ein Bild der Imagination als psychischer Kraft, die in dem Feld der Gefühle nicht nur dazu fähig ist, das schon Gegebene zu reproduzieren, sondern Neues zu erschaffen.

\subsection{Die Gesetze der Abstumpfung und der Beeinflussbarkeit}

Obgleich die Phantasiegefühle gegenüber den genannten intellektuellen Vorgängen einen affektiven Charakter aufweisen, sind sie anders als die echten Gefühle. Um das eigene Terrain der Phantasiegefühle zu bestimmen, untersucht Saxinger, ob beide Phänomene auf denselben Dispositionen gründen. Konkret richtet er seine Aufmerksamkeit auf die Gesetze der Abstumpfung und der Beeinflussbarkeit bei beiden Gefühlsarten.

Wie sind Ernstgefühle einerseits und Phantasiegefühle andererseits dem Gesetz der Abstumpfung unterworfen? Der Zeitverlauf der Ernstgefühle ist dergestalt, dass sie zunächst eine gewisse Intensität erreichen und mit der Zeit abstumpfen. Phantasiegefühle sind dem Gesetz der Abstumpfung dagegen nach Saxinger nicht unterworfen: Sie sind relativ unveränderlich (Saxinger 1904, S. 589). Dies gilt - so meine Interpretation - sowohl für Phantasiegefühle, die in Phantasiewahrnehmungen gründen, als auch für Phantasiegefühle, die auf Annahmen basieren. Als Beispiel für ersteren Fall können wir uns die Reaktion eines Menschen vorstellen, der

13 Dies beobachtet Barry Smith auch im Fall von Schwarz. Vgl. Smith (1994), S. 134. 
immer dasselbe Kunstwerk betrachten muss. Wir können hier vermuten, dass das Lustgefühl, das er am Anfang empfinden mag, bald beeinträchtigt wird. Wenn dieser Mensch sich aber das Kunstwerk bloß vorstellen muss, dann wird das Gefühl nicht verändert - meint zumindest Saxinger. Dasselbe gilt für diejenigen Gefühle, die auf Annahmen gründen. Falls wir einmal in Lebensgefahr waren und wir uns später die damalige Situation vergegenwärtigen, werden wir möglicherweise wieder Angst empfinden. Diese Angst gründet auf der Annahme darüber, was uns damals hätte geschehen können. Es ist eine Annahme, weil wir die Sachlage nicht mehr als gefährlich beurteilen. In letzterem Fall würde das Urteilsgefühl der Angst abgestumpft, für das Phantasiegefühl gilt dies jedoch nicht. Die Trauer über den Verlust eines geliebten Menschen stumpft sich mit der Zeit ab, während das Phantasiegefühl, das in der Annahme gründet, wie schön es wäre, wenn er noch lebte, unverändert bleibt (Saxinger 1904, S. 589; auch in Saxinger 1908, S. 418). Daraus zieht Saxinger den Schluss: „Man kann ein Phantasiegefühl, so oft man will, erzeugen, one eine merkliche Veränderung an demselben wahrzunehmen" (Saxinger 1904, S. 590). Phantasiegefühle stumpfen nicht ab oder tun dies zumindest nicht in derselben Weise wie die Ernstgefühle (Saxinger 1908, S. 419). Allerdings scheint Saxinger mir hier zu übersehen, dass wir uns mit einem Phantasiegefühl übersättigen können, etwa wenn wir es zu oft erzeugen, so dass es dann ebenfalls zu einem Prozess der Abstumpfung kommt.

Können sich Ernst- und Phantasiegefühle gegenseitig beeinflussen? Witasek hat behauptet, dass es im Unlustzustand etwa der Trauer schwierig ist, dass ein lustvolles Gefühl sich einstellt. Ernstgefühle können somit die Erzeugung der Phantasiegefühle beeinflussen. Saxinger dagegen behauptet, dass Ernstgefühle die Entstehung der Phantasiegefühle nicht direkt beeinflussen, sondern die Entstehung der intellektuellen Grundlagen für das Phantasiegefühl. Wenn wir traurig sind, können wir erfreuliche Dinge schwieriger vorstellen oder annehmen. Schaffen wir dies doch, „stellt sich das Phantasiegefühl trotz des herrschenden Gefühles ein“ (Saxinger 1904, S. 592). Daraus schließt Saxinger, dass Phantasiegefühle von den aktuellen Gefühlen nicht beeinflusst werden (Saxinger 1904, S. 592; 1908, S. 421; 1902a, S. 393; 1902b, S. 25). Auch die Einwirkung in der entgegensetzten Richtung findet demnach nicht statt: Phantasiegefühle 
beeinträchtigen in keiner Weise wirkliche Gefühle. Ernst- und Phantasiegefühle folgen darüber hinaus auch untereinander unterschiedlichen Regeln. Wenn mehrere Ernstgefühle zusammen auftreten, beeinflussen sie sich gegenseitig. Dies geschieht aber nicht, wenn Ernst- und Phantasiegefühle gleichzeitig erscheinen: anstatt sich zu beeinflussen, koexistieren sie nebeneinander (Saxinger 1904, S. 592; noch detaillierter dargestellt in Saxinger 1902a, S. 402). Daraus ergibt sich, dass beide Gefühlsarten unterschiedlicher Natur sind. Aber dieser Schluss scheint auch problematisch. Wenn wir uns mitten in einer sehr schwierigen Lebenslage, die uns traurig macht, vorstellen, bald in einer besseren Lage zu sein und uns darauf freuen, scheint mir diese Freude das tatsächliche Gefühl der Trauer zu beeinflussen.

\subsection{Gefühlssuggestionen und Phantasiegefühle}

Eine weitere These Witaseks, die Saxinger einer Prüfung unterziehen möchte, betrifft den Begriff der „Gefühlssuggestion“. Witasek zufolge sind die Gefühlssuggestionen ein wichtiger Bestandteil der Phantasiegefühle. Demgegenüber behauptet Saxinger im Rahmen einer umfangreichen Untersuchung der Suggestion im Allgemeinen, dass Gefühlssuggestionen im eigentlichen Sinne des Wortes nicht möglich sind (Saxinger 1908, S. 406).

Saxingers These lautet, dass bei dem Phänomen, das wir als Gefühlssuggestion bezeichnen, tatsächlich nicht das Gefühl selbst „suggeriert“ wird, sondern die Disposition zu demselben. Es gibt demnach keine Gefühlssuggestionen im eigentlichen Sinne des Wortes, sondern Suggestionen zu den Dispositionen für Gefühle. Laut Saxinger können wir ein Gefühl erzeugen, indem wir die für eine Emotion nötigen intellektuellen Grundlagen durch Suggestion hervorrufen, so dass sich dann die entsprechende Gefühlsregung einstellt (Saxinger 1908, S. 406). In anderen Fällen geschieht dies auch durch die Körperhaltung oder Mimik, die für ein bestimmtes Gefühl typisch ist. ${ }^{14}$ Gefühle können durch Suggestion ,erzeugt,

14 Im damaligen Zusammenhang wurde diese Tatsache auch von James und Stumpf beobachtet (vgl. James 1905; Stumpf 1928). 
verändert und zum Verschwinden gebracht werden können“" (Ebd., S. 407), nur insofern wir die Dispositionen zu diesen Gefühlen - und nicht die Gefühle selbst, wie Witasek behauptet hat - neu schaffen, verändern und vernichten.

Trotz dieser Kritik an Witasek teilt Saxinger auch zwei Thesen mit diesem Autor. Zum einem sind beide der Ansicht, dass Gefühlssuggestionen und Ernstgefühle unterschiedlicher Natur sind. Gegenüber Ernstgefühlen sind „suggerierte“ Gefühle in der Regel nicht von Dauer und machen bei ihrem Verschwinden wieder Platz für das ursprüngliche Gefühl (Saxinger 1908, S. 408). Zum anderen ist beiden die These gemeinsam, dass Gefühlssuggestionen bei der Bildung von Phantasiegefühlen eine wichtige Rolle spielen. Denn die anschauliche Vorführung der gedachten Gefühle bzw. ihrer intellektuellen Grundlagen in der Phantasie ermöglicht die Entstehung von Phantasiegefühlen und fungiert sogar als Brücke zur Erzeugung der eigentlichen Gefühle (Saxinger 1908, S. 413).

\subsection{Phantasiegefühle als Annahmegefühle? Die Möglichkeit von Phantasievorstellungsgefühlen}

Eine der kontroversen Thesen Witaseks in der Auseinandersetzung mit Meinong war die, dass Phantasiegefühle Annahmegefühle seien und deswegen kein eigenständiges Phänomen. Ernst Schwarz wird - so meine Interpretation - diese These zugunsten von Meinongs Position in „Über Phantasiegefühle“ bestreiten, indem er erstens die Existenz von „Phantasievorstellungsgefühlen“ - d.h. Gefühlen, welche in PhantasieWahrnehmungen und Erinnerungen gründen - nachweist und zweitens für die Eigenständigkeit der Klasse der Phantasiegefühle gegenüber ihren „ernsten“ Gegenstücken plädiert.

Wie unterscheiden sich Phantasievorstellungsgefühle von Vorstellungsgefühlen? Wie unterscheidet sich die Angst angesichts einer phantasierten Wahrnehmung oder Erinnerung von der Angst angesichts einer echten Wahrnehmung?

Beide Gefühlsarten unterscheiden sich zunächst hinsichtlich ihrer kognitiven Basis: Vorstellungsgefühle gründen auf Wahrnehmungen wäh- 
rend Phantasievorstellungs-gefühle auf Phantasie-Wahrnehmungen und Erinnerungen basieren.

Schwarz postuliert aber auch Unterschiede hinsichtlich der Intensität. Er zeigt anhand von Experimenten auf dem Gebiet der Tast-, Geruchsund Geschmacksempfindungen, dass Phantasievorstellungsgefühle eine geringere Intensität als Vorstellungsgefühle haben (Schwarz 1906a, S. 486). Die Intensität der Phantasievorstellungsgefühle hängt hauptsächlich von drei Faktoren ab. Sie hängt an erster Stelle von der Intensität ihrer Voraussetzungen ab: Je schwächer eine Vorstellung ist, desto weniger lebhaft wird das Phantasiegefühl sein, das auf dieser Vorstellung gründet. Laut Schwarz überträgt sich die „Blässe und Körperlosigkeit“ der Phantasiewahrnehmungen und Erinnerungen auf die Phantasievorstellungsgefühle, so dass es eine gewisse Einförmigkeit der Intensitäten bei den Phantasiegefühlen gegenüber der Mannigfaltigkeit der Intensitäten bei Ernstgefühlen gibt (Schwarz 1906b, S. 95). Die Intensität der Phantasievorstellungsgefühle hängt an zweiter Stelle von den korrespondierenden Ernstgefühlen ab: Je stärker das Ernstgefühl gewesen wäre, desto lebhafter müsse das zugeordnete Phantasiegefühl sein (Ebd., S. 96). Schließlich hängt die Intensität der Phantasiegefühle auch von den anderen vorhandenen Gefühlen ab: Sie kann gehemmt oder verstärkt werden. In diesem letzten Aspekt der Beeinflussung unterscheidet sich die Ansicht von Schwarz von der vorher genannten Ansicht Saxingers, wonach beide Gefühlsarten sich nicht beeinflussen können.

Ein dritter Unterschied beider Gefühlsarten betrifft ihre Verlaufsform. Das Auftauchen und Verschwinden von Phantasievorstellungsgefühlen ist ähnlich wie das Kommen und Gehen von Phantasievorstellungen: Sie kommen und gehen sehr schnell und sind in den psychischen Zusammenhang nicht fest eingebettet (Schwarz 1906b, S. 85; 1906a, S. 487). Dieser Unterschied lässt sich in der inneren Beobachtung bestätigen. Während es schwierig ist, die Qualität, Intensität und Verlaufsform der Ernstgefühle wahrzunehmen und diese im Bewusstsein zu isolieren, hebt sich im Bewusstsein das Phantasiegefühl „klar und deutlich von dem übrigen Gefühlshintergrunde ab" (Schwarz 1906b, S. 101; 1906a, S. 487). Deshalb sind Phantasiegefühle auch stärker unserem Willen unterworfen. 
Weitere Unterschiede betreffen das so genannte Sukzessionsverhältnis beider Gefühlsarten. Schwarz untersucht hier eine wichtige These Wundts. Wundt behauptet, dass bei Ernstgefühlen zunächst die intellektuelle Basis vorhanden ist und dann das Gefühl erzeugt wird, während bei Phantasievorstellungsgefühlen das Gefühl der Vorstellung vorangeht. Dies ist so, weil die Reproduktion einer Erinnerung mehr Zeit benötigt als die Erzeugung einer direkten Wahrnehmung und weil die Erinnerungen im Bewusstsein länger unbemerkt bleiben, so dass sie sich zunächst sammeln und verdichten müssen, um wahrgenommen zu werden. Schwarz stimmt dieser These zu, stellt allerdings auch fest, dass Phantasievorstellung und Gefühl auch gleichzeitig auftreten können (Schwarz 1906a, S. 489).

Eine weitere Abweichung zwischen beiden Gefühlsarten betrifft ihre psychische Wirksamkeit. Nach der experimentellen Untersuchung der Gefühle im Gebiet der Bewegungs-, Tast- und Schmerzempfindungen kommt Schwarz zu dem Schluss, dass, während Ernstgefühle mit anderen Ernstgefühlen einen Komplex bilden und sich zu einem Gesamteffekt summieren, dies bei den Phantasievorstellungen und den auf ihnen gründenden Gefühlen nicht der Fall ist (Schwarz 1906a, S. 494-495). Wenn man etwa viel über eine bestimmte Krankheit liest oder sich die Anstrengung eines Marathonlaufs vor Augen führt, sind diese Vorstellungen gefühlsbetont, aber wir erleiden die damit bezogenen Gefühle nicht.

Schließlich gibt es auch Unterschiede, was die Gesetzlichkeit gegenüber Dispositionen anbelangt. Können Phantasiegefühle nur dann erlebt werden, wenn die zugeordneten Ernstgefühle schon einmal erfahren worden sind? Oder können sich Phantasiegefühle auch dort einstellen, wo nie zuvor ähnliche Ernstgefühle erlebt worden sind? Schwarz ist der Meinung, ,dass Phantasiegefühle auch dann aktualisiert werden können, wenn ihnen die zugeordneten Ernstgefühle nicht vorangegangen sind“" (Schwarz 1906b, S. 88). Dies geschieht selbst dann, wenn die zugrundeliegenden Annahmen uns unwahrscheinlich erscheinen. Auf den Bereich etwa der Literatur bezogen, bedeutet dies, dass wir ausgehend von literarischen Fiktionen unseren Gefühlshorizont erweitern können, weil uns Erfahrungen nahegebracht werden, die wir sonst vielleicht nie machen würden. Allerdings ist es hier wichtig zu betonen, dass trotz dieser Mög- 
lichkeit der Erweiterung unserer Gefühlspalette Phantasiegefühle weder die Fähigkeit haben, Dispositionen von Vorstellungen zu verstärken noch Dispositionen zu Ernstgefühlen herabzusetzen (Ebd., S. 101). D.h., dass wir dank der Literatur bestimmte Erfahrungen machen können, aber dass diese neuen Erlebnisse in uns keine psychischen Dispositionen begründen. Ernstgefühle dagegen können unter Umständen psychische Dispositionen implementieren (Ebd., S. 90).

Schwarz' ausführliche Untersuchung richtet sich in zweierlei Hinsicht gegen Witasek. Phantasiegefühle sind nicht automatisch als Annahmegefühle zu verstehen, da es auch Phantasiegefühle gibt, die in Phantasievorstellungen gründen. Es ist daher kein Kennzeichen der Phantasiegefühle, dass sie sich auf Fiktives richten, da die Klasse der Phantasiegefühle auch diejenigen Gefühle umfasst, die sich auf Nichtaktuelles wie Phantasiewahrnehmungen und Erinnerungen richten. Zum anderen zeigt Schwarz, dass die Phantasiegefühle eine eigene Klasse psychischer Phänomenen sind.

\subsection{Phantasiegefühle: Ein eigenständiges Phänomen zwischen Vorstellung und Gefühl}

Phantasiegefühle bilden laut Saxinger und Schwarz eine eigene Grundklasse psychischer Phänomene - Phantasiegefühle sind ein emotionales Phänomen sui generis (Saxinger 1908, S. 426). Im Einklang mit Meinong vertritt Saxinger die These, dass den Phantasiegefühlen eine Zwischenstellung zwischen Vorstellungen und Gefühlen zukommt (Meinong 1977, S. 233, Saxinger 1908, S. 411). Gegen Witasek behauptet er, dass Phantasiegefühle nicht auf bloße Annahmegefühle reduziert werden können, da sie auf Vorstellungen, Annahmen und sogar Urteilen gründen können (Saxinger 1904, S. 602). Dasselbe gilt auch für die Phantasiebegehrungen, da auch wirkliche Begehrungen Annahmen als Voraussetzungen haben können (Saxinger 1904, S. 603). Es gibt ferner einen Unterschied zwischen Phantasiegefühlen und Ernstgefühlen, der den „emotionalen Faktor“ betrifft, da beide - wie schon Hume und Meinong bemerken - verschieden gefühlt werden (Saxinger 1904, S. 602; auch Saxinger 1908, S. 424). 
Ist diese Aufstellung einer neuen psychischen Kategorie nötig? Saxingers These lautet, dass die genannten Phänomene eine „stellvertretende Funktion" haben. Sie treten dort auf, wo es keine wirklichen Emotionen gibt. Sie sind zweckmäßige psychische Tatsachen, weil durch sie Willensenergie im Vergleich zu den wirklichen Gefühlen gespart wird (Saxinger 1904, S. 606).

Auch für Schwarz stehen die Phantasiegefühle ,,in der Mitte zwischen Vorstellungen und Gefühlen“ (Schwarz 1906b, S. 84). Phantasiegefühle ähneln den Vorstellungen in folgenden Merkmalen: dem Verlauf, der klaren Abgrenzbarkeit von ihrem psychischen Hintergrund und der Unfähigkeit, Gefühlsdispositionen zu festigen oder zu schwächen. Gleichzeitig aber ähneln sie den Ernstgefühlen, weil sie zwischen Lust und Unlust schwanken, intellektuelle Voraussetzungen benötigen, im Gegenpaar mit Ernstgefühlen auftreten und durch das Vorhandensein von anderen Gefühlen gefördert oder beeinträchtigt werden. Es gibt also Ähnlichkeiten zu beiden Arten psychischer Akte, ohne dass die Phantasiegefühle ihre eigene Realität als emotionale Tatsachen verlieren (Schwarz 1906b, S. 103).

\section{Quasi-Gefühle in der analytischen Philosophie \\ 6.1 Die Lehre der Scheingefühle und das Paradoxon der Fiktion}

Im Rahmen der Debatte über „Das Paradoxon der Fiktion“ wurde den Gefühlen über Fiktionen in der analytischen Philosophie besondere Aufmerksamkeit zuteil. Obgleich zwischen den neuen analytischen Ansätzen und der älteren Lehre der Scheingefühle Unterschiede bestehen, teilen beide Traditionen ähnliche Thesen und haben gemeinsame Forschungsstränge. Ein Vergleich beider Debatten kann daher möglicherweise neue Perspektiven eröffnen.

Blicken wir zunächst auf die Unterschiede. An erster Stelle ist der Entstehungsrahmen beider Debatten verschieden. Während die Untersuchung der Scheingefühle auf eine Beschreibung der ästhetischen Erfahrung und der Einstellungsänderung des Subjekts beim Konsum von Kunst 
abzielt, geht es in der jüngeren Diskussion eher darum, die Frage nach der Rationalität der Gefühle zu beantworten. Es besteht heute kein Konsens über den Begriff der ästhetischen Einstellung, vielmehr wird gelegentlich sogar die Möglichkeit derselben bestritten (etwa Dickie 1964). Gefühle gegenüber Fiktionen stehen im Zentrum des heutigen analytischen Interesses, weil sie eine „Herausforderung“ der doxastischen und der praktischen Rationalität sind. Im heutigen kognitivistischen Verständnis der Gefühle, wo diese wesentlich auf Urteilen gründen, wird es als irritierende Tatsache registriert, dass es Emotionen geben soll, die nicht auf Urteilen gründen und insbesondere kein Existenzurteil über ihre Objekte beinhalten. Darüber hinaus ist in der aktuellen Debatte die These dominant, dass Gefühle zum Handeln motivieren. Da fiktionalen Gefühlen oft der Link zur Motivation fehlt, werden sie als suspekt behandelt. Aufgrund der Herausforderung an beiderlei „Rationalitäten“ - die doxastische und die praktische - werden fiktionale Gefühle als ,paradox“ angesehen.

Weitere Unterschiede betreffen den Umfang der Fragestellung beider Debatten. Während sich die Untersuchung der Scheingefühle für die imaginative Einstellung und die möglichen Wechselwirkungen der Phantasie von Autor, Schauspieler, Zuschauer und Leser interessiert, steht im Mittelpunkt der heutigen Debatte fast ausschließlich die Leser- und Zuschauerperspektive. Somit wird der Raum der Phantasie zumindest in der Forschung begrenzt. Ferner war die alte Lehre der Scheingefühle daran interessiert, eine Erklärung der ästhetischen Gefühle hinsichtlich der Kunst insgesamt zu finden, während sich die heutige philosophische Debatte auf drei Gattungen begrenzt: Literatur, Theater und Film. Die Gefühle bei Musik bilden ein eigenes Forschungsfeld, die hier entwickelten Theorien finden aus vielleicht naheliegenden Gründen keinen Eingang in die Diskussion, die unter dem Stichwort „Paradoxon der Fiktion“ stattfindet.

Gemeinsam sind beiden Debatten ein kognitivistisches Bild der Gefühle, bei dem den Urteilen eine besondere Rolle für das Gefühlsleben zukommt, und die Auffassung der Gefühle als Motivationsfaktoren. In beiden Traditionen zeigt sich ferner eine Unterordnung der Welt der Fiktion und des Spiels als Akt der Imagination unter die Wirklichkeit und die ernsthaften Akte wie den Urteilen. Aufgrund dieser Gemeinsamkeiten gibt es mehrere Forschungsstränge, die in beiden Debatten zu finden sind. 


\subsection{Quasi-Gefühle}

Eine der gemeinsamen Forschungsachsen beider Debatten betrifft die These der Quasi-Gefühle. In der analytischen Tradition finden wir die These bei Ryle, Kenny, Budd, Mulligan und Walton in einer sehr ähnlichen Form wie bei den Autoren der Grazer Schule vertreten. Als einziger unter den heutigen Autoren hat Kevin Mulligan in direktem Anschluss an die österreichischen Philosophen einen eigenen Beitrag über die QuasiGefühle geleistet. Mulligan bezeichnet als Quasi-Gefühle solche Gefühle, die auf Annahmen, Erinnerungen und Einfühlungsakten gründen (Mulligan 1992). Ihm zufolge sind die Quasi-Gefühle dem Willen unterworfen, während sich reale Gefühle nicht leicht manipulieren lassen.

Unabhängig von den Autoren der Grazer Schule entwickelt Walton in Mimesis as Make-Believe (1990) eine fiktionalistische Theorie der QuasiGefühle. Walton geht in seinem Beitrag von der Unterscheidung zwischen Realität und Fiktion und entsprechenden Urteilen und Gefühlen aus. Die Urteile über die Realität sind Urteile erster Ordnung, die Urteile über die Fiktion - etwa „Anna Karenina ist unglücklich verheiratet“ - bezeichnet er als Urteile zweiter Ordnung oder Make-Believe. Diese Unterscheidung zwischen zwei Arten von Urteilen bedingt eine Unterscheidung zwischen zwei Arten von Emotionen, die auf den verschiedenen Urteilen gründen sollen. Die Emotionen des realen Lebens gründen auf Urteilen erster Ordnung und sind reale Emotionen. Die Emotionen der Fiktion gründen hingegen auf Urteilen zweiter Ordnung oder auf „make-believe“ und sind laut Walton „Make-believe“-Emotionen oder „Quasi-Emotionen“. QuasiGefühle haben laut Walton dieselbe Struktur wie die Gefühle, die bei Kinderspielen („make-believe games“) entstehen. Man tut, als habe man ein Gefühl, obgleich keine echte Emotion vorhanden ist. Mich interessiert hier nicht die mögliche Kritik an Waltons Analogie zwischen Gefühlen bei Kinderspielen und Gefühlen gegenüber Kunstwerken, sondern bloß die Tatsache, dass Walton - genau wie vor ihm Meinong - Kunst als eine fortgeschrittene Form von Spiel versteht und dass er den Gefühlen, die sich darauf richten, den Status des Echten abspricht. Quasi-Gefühle sind laut Walton phänomenal den echten Emotionen gleich, können aber nicht als Gefühle bezeichnet werden, weil sie auf Make-Believe gründen und 
ihnen eine motivationale Kraft fehlt (Walton 1993, S. 196, 202, 255 und 271).

\subsection{Der Schatten der Irrationalität und das kognitivistische Model}

Gefühle über Fiktionen werden in der heutigen Debatte als Herausforderung der doxastischen und der praktischen Rationalität betrachtet. Sie sind eine Herausforderung der doxastischen Rationalität, weil die meisten Autoren die Gefühle über deren kognitive Aspekte definieren. So haben etwa Solomon und Nussbaum die Gefühle als eine Art von Urteilen aufgefasst (Solomon 1993; Nussbaum 2005), Marks und Green verstehen die Gefühle als „Belief-Desire“-Komplexe (Green 1992) und Ben-ze’ev hat eine Mehrkomponententheorie der Gefühle entwickelt, bei der diese eine $\mathrm{Zu}-$ sammensetzung von Urteilen und anderen Elemente wie Empfindungen, Motivationen und Kognitionen sind (Ben-ze'ev 2000). Auch in denjenigen Modellen, wo den Emotionen eine eigene Realität zugeschrieben wird und keine Reduzierbarkeit auf Urteile, werden die Urteile als notwendige Wesenselemente angesehen. Kenny und Taylor postulieren etwa, dass die Gefühle ausschließlich auf Urteilen gründen (Kenny 1963; Taylor 1985). Vor diesem kognitivistischen Hintergrund werden diejenigen Gefühle, die wie die Gefühle über Fiktionen nicht auf Urteilen gründen, als problematisch behandelt. Colin Radford bezeichnet etwa fiktionale Emotionen als irrational (Radford 1975, 1982 und 1995). Es wird im Rahmen des Kognitivismus unplausibel betrachtet, dass man eine Emotion über etwas haben können soll, um dessen Nichtexistenz man wie im Fall einer literarischen Figur weiß.

Sollen Gefühle über Fiktionen nicht im Widerspruch zur doxastischen Rationalität stehen, bietet es sich ein, eine schwächere Fassung von Kognitivismus hinsichtlich der Emotionen zu vertreten. In der heutigen Debatte gibt es eine Minderheit von Autoren, die ein Bild der Emotionen anbietet, bei dem nicht nur Urteile als intellektuelle Basis gelten, sondern auch andere Elemente wie Wahrnehmungen, Phantasien, Erinnerungen, Erwartungen und Annahmen. Hierbei sind Greenspan, de Sousa, Stocker, 
Mulligan, Elster, Goldie und Tappolet zu erwähnen (Greenspan 1980; de Sousa 1987; Stocker 1987; Mulligan 1998; Elster 1999; Goldie 2002; Tappolet 2000). Nur wenn wir diese erweiterten Modelle annehmen, können wir die Gefühle, die nicht in Urteilen gründen, in das Paradigma der Rationalität einbeziehen. Im Rahmen der Ästhetik haben Matravers, Gendler und Kovakovich ebenfalls postuliert, dass fiktionale Emotionen nicht unbedingt auf Urteilen gründen (Matravers 2006, S. 254; Gendler \& Kovakovich 2006, S. 251-252). Diese Autoren haben aber die Frage nach den kognitiven Grundlagen der Gefühle nicht weiter untersucht. Für andere Autoren etwa Weston und Lamarque gründen fiktionale Emotionen in Gedanken anstatt in Urteilen (Weston 1975; Lamarque 1981). Die fiktionalistische Lösung Waltons ist in diesem Rahmen ein Forschritt, da er anerkennt, dass fiktionale Emotionen nicht in Urteilen gründen und daher auch nicht sinnvollerweise als irrational zu bezeichnen sind. Auch die Grazer Theorie der Quasi-Gefühle basiert auf einer kognitivistische Konzeption der Gefühle, wonach diese in ernsthaften Akte und insbesondere in Urteilen gründen müssen, um den Namen Gefühl zu verdienen. Wir sehen insofern, dass in beiden Schulen mit einem sehr normativen Begriff des Gefühls gearbeitet wird. Meinongs Ansatz über die Quasi-Gefühle als Gefühle, die in Annahmen gründen, ähnelt Waltons Beitrag über MakeBelieve Emotionen.

Was die praktische Rationalität anbelangt, wird in beiden Traditionen mit der Annahme gearbeitet, dass Gefühle Handlungen motivieren, während Quasi-Gefühlen der Link zur Motivation fehlt. Die Autoren der Grazer Schule sprechen von Phantasie-Begehrungen, um die Wünsche zu bezeichnen, welche in uns entstehen, wenn wir uns mit Fiktionen auseinandersetzen. Die heutigen analytischen Autoren gehen ebenfalls davon aus, dass Quasi-Gefühle eine Spannung zur praktischen Rationalität zeigen, weil sie keine Handlungen motivieren (etwa Radford 1975; Walton 1990). Eine sehr ähnliche These wie die These der Phantasie-Begehrungen ist bei Currie, Egan und Doggett zu finden: sie sprechen von ,i-desires“ oder „narrative desires“ (Currie 2002; Dogget \& Egan 2007).

Gegen die Annahme, dass Gefühle immer zu Handlungen motivieren und Quasi-Gefühlen diese Eigenschaft fehlt, haben Moran und Goldie erstens gezeigt, dass nicht alle Gefühle über Wirkliches unbedingt zum 
Handeln motivieren, und zweitens, dass nicht alle fiktionalen Emotionen die Verbindung zur Motivation fehlt (Moran 1994; Goldie 2003). Auch Gefühle über Fiktionen veranlassen uns zum Handeln, obgleich dies in der Regel auf eine indirekte und weniger deutliche Weise geschieht.

\subsection{Einfühlung und das Paradoxon des Tragödie}

Es gibt weitere Aspekte, welche in beiden Schulen zu finden sind. Es handelt sich zunächst um die Frage nach der Teilnahme am Schicksal fiktionaler Figuren. Die heutige analytische Philosophie spricht von Einfühlung bzw. Empathie. Wollheim unterscheidet zwischen verschiedenen Formen, sich in fiktionale Situationen zu versetzen, nämlich einem zentralen und einem azentralen Imaginieren. Ersteres geschieht, wenn ich einen bestimmten Blickwinkel annehme, letzteres wenn ich eine Situation phantasiere ohne eine bestimmte Perspektive anzunehmen (Wollheim 1984). Goldie hat diese Begriffe für fiktionale Charaktere weiterentwickelt (Goldie 2003). Neben der Einfühlung ist auch von „Projektion“ die Rede. Diese wurde von Gholamain und Oatley für fiktionale Figuren ausgiebig untersucht (Gholamain \& Oatley 1997). In der früheren Debatte über Scheingefühle wurde der Begriff von Lipps und Witasek ausführlich dargestellt. Der Vergleich beider Traditionen zeigt, dass der heutigen Debatte eine genauere Unterscheidung bestimmter Formen der Anteilnahme an fiktionalen Charakteren immer noch fehlt.

Im Zusammenhang damit steht die Frage, warum wir unlustvolle Gefühle empfinden wollen, wenn wir uns mit Fiktionen engagieren. Diese Thematik wurde von Hume als Paradoxon der Tragödie bezeichnet, die Autoren der Scheingefühle haben sich ebenfalls dafür interessiert, und heute wird die These unter dem Stichwort „Paradoxon des Horrors“ untersucht (Yanal 1999; Feagin 2004 u.a.) 


\subsection{Der kognitive Wert der Literatur}

In beiden Schulen steht auch die Frage nach der Funktionalität von Gefühlen über Fiktionen im Mittelpunkt. Für die Grazer Autoren haben Quasi-Gefühle eine stellvertretende Funktion und sie zielen darauf, psychische Anstrengungen zu ersparen. Heute schreiben Susan Feagin, Tamar Szabó Gendler und Karson Kovakovich und ganz besonders Richard Rorty und Martha Nussbaum den fiktionalen Emotionen eine instrumentelle Rolle zu (Rorty 2003; Nussbaum 1990 und 1997). Fiktionale Emotionen helfen uns, einen Blick für andere mögliche Leben zu haben, und dank ihnen können wir Situationen erleben, die uns sonst niemals zugänglich wären. Wir erweitern so unser Repertoire an Gefühlsreaktionen, unsere wenn man so will - emotionale Palette. Damit wird den Quasi-Gefühlen nicht nur eine kognitive Bedeutung zugeschrieben, sie werden auch als moralisch prägend bezeichnet. Dies soll freilich nicht so verstanden werden, als ob wir mit dem Ziel einer solchen Bereicherung fiktionale Emotionen haben und haben wollen. Man kann in Abrede stellen, dass wir etwa einen Roman derart instrumentell lesen (Nussbaum 1990; Matravers 2006, S. 260). Fiktionen, so wie wir Menschen sie erfinden, und die Emotionen, die sie in uns erwecken, können vielmehr einen intrinsischen Wert haben, sie sind Selbstzweck. Wir lesen einen Roman und schauen einen Film, weil uns dies Genuss bereitet.

In den genannten Beiträgen stecken zwei wichtige Thesen. Erstens findet sich hier implizit die These, dass man in der Imagination das erleben kann, was man vorher nicht erlebt hat. Diese These steht diametral gegen Kant und Jung, für welche man in der Phantasie nur das erleben kann, was man schon kennt. Auch diese These ist bei den Grazer Autoren $\mathrm{zu}$ finden.

Zweitens findet sich hier die These, dass das, was in der Imagination erlebt wird, nicht an der Oberfläche bleibt, sondern die Fähigkeit besitzt, psychische Dispositionen zu begründen. Die Grazer Autoren hatten noch das Gegenteil behauptet.

Es gibt andere Thesen der Lehre der Scheingefühle, welche keine Parallelen in den heutigen Debatten finden oder wenig Aufmerksamkeit erhalten haben. Es sind unter anderem die von der leiblichen Andersartig- 
keit der Quasi-Gefühle im Vergleich zu echten, die Idee der Gefühlssuggestionen, das Postulat der psychischen Resonanz und der Gefühlsgestalten, die Relevanz des ästhetischen Genusses als einzigem echten ästhetischen Gefühl und die Rolle der Imagination als transformative Kraft psychischer Akte. In der heutigen philosophischen Debatte fehlt auch der Einbezug der Perspektive des Autors und Schauspielers. Letztlich gibt es auch kein Äquivalent zu dem ambitionierten Projekt, eine Theorie der Scheingefühle zu entwickeln, die gültig für alle Künste wäre.

Sind Gefühle über Fiktionen echte Gefühle oder bloß Quasi-Gefühle? Darüber besteht heute genauso wenig Einigkeit wie vor hundert Jahren. Ob wir ein Fühlen aus dem Reich der Gefühle verbannen müssen, weil es nicht in Urteilen gründet, oder ob fiktionale Gefühle eine Unterarte ,echter" Gefühlen sind, bleibt kontrovers. Die Antwort hängt stark von unserem operativen Gefühlsbegriff und unserer persönlichen Erfahrung von Kunst ab. Aber es bleibt die Frage, warum wir aus der Mannigfaltigkeit unseres Gefühlslebens ein bestimmtes Phänomen, das für unsere Erfahrung von Kunst von entscheidender Bedeutung ist, ausschließen sollen? Wie Lipps es ausgedrückt hat: „Es gibt eben nicht nur zwischen Himmel und Erde allerlei, (...) sondern es gibt auch in des Menschen Brust noch allerlei, insbesondere allerlei Weisen sich zu fühlen“ (Lipps 1905, S. 480). ${ }^{15}$

\section{Íngrid Vendrell Ferran}

CISA - Université de Genève

Ingrid.Vendrell@unige.ch

15 Für Anregungen zu diesem Aufsatz möchte ich mich bei Christoph Johanssen, Kevin Mulligan, Arito Rüdiger Sakai und Arto Haapala bedanken. Dieser Aufsatz wurde im Rahmen des Projektes „Gefühl, Phantasie und Fiktion“ am Centre Interfacultaire de Sciences Affectives (Université de Genève) geschrieben, das von der Deutschen Forschungsgemeinschaft finanziert wird. 


\section{Literatur}

Barbero, Carola (2007), "Crocodile Tears", in Latin Meeting in Analytic Philosophy Genova 20-22 September 2007 Proceedings. S. 93-102.

Ben-ze'ev, Aaron (2000), The Subtlety of Emotions, Massachusetts, MIT Press.

Budd, Malcom (1985), Music and the Emotions: The Philosophical Theories, London, Routledge.

Currie, Gregory (2002), "Imagination as Motivation", Proceedings of the Aristotelian Society, 102 (3), S. 201-216.

Currie, Gregory \& Ravenscroft, Ian (2002), Recreative Minds, Oxford, Oxford University Press.

de Sousa, Ronald (1987), The Rationality of Emotion, Cambridge, Cambridge Macs.

Dickie, George (1964), "The Myth of the Aesthetic Attitude", American Philosophical Quarterly, 1, 1, S. 56-66.

Doggett, Tyler \& Egan, Andy (2007), "Wanting Things You Don't Want: The Case for an Imaginative Analogue of Desire", Philosophers' Imprint, 7 (9), S. 1-17.

Dorner, Leo (1975), “Zu den Grundproblemen der Musikästhetik”, Österreichische Musikzeitschrift, 8, S. 393-400.

Elster, Jon (1999), Alchemies of the Mind, New York, Cambridge University Press.

Feagin, Susan L. (2004), "The Pleasures of Tragedy", in E. John \& D. McIver Lopes [eds.] (2004), S. 185-194.

Geiger, Moritz (1914), "Das Problem der ästhetischen Scheingefühlen", in Bericht des Kongresses für Ästhetik und allg. Kunstwissenschaft. Berlin 7-9. Okt. 1913, Stuttgart, Enke.

Geiger, Moritz (1974), Beiträge zur Phänomenologie des ästhetischen Genusses, Tübingen, Niemeyer. 
Gendler, Tamar S. \& Kovakovich, Karson (2006), "Genuine Rational Fictional Emotions", in M. Kieran [ed.] (2006), S. 241-254.

Gholamain, Mitra \& Oatley, Keith, (1997), „Emotions and Identification: Connections between Readers and Fiction“, in M. Hjort. \& S. Laver [eds.] (1997), Emotion and the Arts, Oxford, Oxford University Press, S. 263-282.

Goldie, Peter (2002), The Emotions. A Philosophical Exploration, Oxford, Clarendon Press.

Goldie, Peter (2003), „Narrative, Emotion and Perspective“, in M. Kieran \& D. McIver Lopes [eds.] (2003), Imagination, Philosophy, and the Arts. London, Routledge, S. 54-69.

Green, O. H. (1992), The Emotions, Dordrecht / Boston / London, Kluwer.

Greenspan, Patricia S. (1980), "A case of mixed feelings: ambivalence and the logic of emotion", in A. O. Rorty [ed.] (1980), Explaining Emotions, Berkeley und Los Angeles, University of California Press, S. 223-250.

Gross, Karl (1902), Der ästhetische Genuß, Giessen, J. Ricker.

Haas, Willy (1910), Über Echtheit und Unechtheit von Gefühlen, Nürnberg, Benedikt Hilz.

Harris, Paul L. (2000), The Work of the Imagination. Understanding Children's Worlds, Oxford, Blackwell Publishing.

Huemer, Wolfgang (2009), "Austrian Aesthetics between Psychology and Psychologism", in B. Centi \& W. Huemer [eds.] (2009), Values and Ontology, Frankfurt et al., Ontos Verlag, S. 267-288.

Hume, David (2004), "Of Tragedy”, in E. John \& D. McIver Lopes [eds.] (2004), S. 69-87.

Hume, David (2008), A Treatise of Human Nature, Oxford, Oxford Philosophical Texts.

James, William (1905), The Principles of Psychology, Bd. II, New York, Henry Holt and Company. 
Kenny, Anthony (1963), Action, Emotion and Will, London, Routledge \& Paul.

Kieran, Matthew [ed.] (2006), Aesthetics and the Philosophy of Art, Oxford, Blackwell.

Kühn, Manfred (2006), „The reception of Hume in Germany“, in P. Jones [ed.] (2006), The Reception of David Hume in Europe, London und New York, Continuum, S. 98-99.

Külpe, Oswald (1921), Grundlagen der Aesthetik, Leipzig, Hirzel.

Lamarque, Peter (1981), "How can we Fear and Pity Fictions?", British Journal of Aesthetics, XXI, S. 291-304.

Lange, Konrad (1901), Das Wesen der Kunst, Bd. I, Berlin, Grote.

Lipps, Theodor (1905), "Weiteres zur Einfühlung”, Archiv für die gesamte Psychologie, IV, S. 465-519.

Matravers, Derek (2006), "The Challenge of Irrationalism, and How Not To Meet It", in M. Kieran [ed.] (2006), S. 254-265

John, Eileen \& McIver Lopes, Dominic [eds.] (2004), Philosophy of Literature. Contemporary and Classic Readings, Oxford, Blackwell.

Meinong, Alexius $\left(1902 / 1910^{2}\right)$, Über Annahmen, Nachdr. in Alexius Meinong Gesamtausgabe, hrsg. von R. Haller und R. Kindinger gemeinsam mit R. M. Chisholm, Bd. IV, Graz, Akademische Druckund Verlagsanstalt, 1977.

Meinong, Alexius (1923), “A. Meinong”, in R. Schmidt [ed.] (1923), Philosophie der Gegenwart, Bd. I, Leipzig, Meiner, S. 100-158.

Moran, Richard (1994), „The Expression of Feeling in Imagination“, The Philosophical Review, 103 (1), S. 75-106.

Mulligan, Kevin (1998), "From Appropriate Emotions to Values", The Monist, 81 (1), S. 161-188.

Mulligan, Kevin (1999), "La varietà e l'unità dell'immaginazione”, Rivista di estetica, n.s. XL, 2, S. 53-67. 
Mulligan, Kevin (2009), "Was sind und was sollen die unechten Gefühle?", in U. Amrein [Hrsg.] (2009), Das Authentische. Zur Konstruktion von Wahrheit in der säkularen Welt, Zürich, Chronos.

Nussbaum, Martha C. (1990), Love's Knowledge. Essays on Philosophy and Literature, Oxford, Oxford University Press.

Nussbaum, Martha C. (1997), Poetic Justice. The Literary Imagination and Public Life, Boston, Beacon Press.

Nussbaum, Martha C. (2005), The Upheavals of Thought. The Intelligence of Emotions, Cambridge, Cambridge University Press.

Ortega y Gasset, José (1954), "Vitalität, Seele, Geist", in Gesammelte Werke, Bd. 1, Stuttgart, Deutsche Verlags-Anstalt, S. 317-350.

Ortega y Gasset, José (2005), "La deshumanización del arte", in Obras Completas III, Madrid, Taurus.

Pfänder, Alexander (1922), Zur Psychologie der Gesinnungen, Halle, Max Niemeyer.

Pugmire, David (1994), "Real Emotions", Philosophy and Phenomenological Research, 54 (1), S. 105-122.

Pugmire, David (2005), Sound sentiments: Integrity in the Emotion, Oxford, Oxford University Press.

Radford, Colin (1975), "How Can We Be Moved by the Fate of Anna Karenina?", Proceedings of the Aristotelian Society, Supplementary Volume 49, S. 67-80.

Radford, Colin (1982), "Philosophers and Their Monstrous Thoughts", British Journal of Aesthetics, 22, S. 261-263.

Radford, Colin (1995), "Fiction, Pity, Fear and Jealousy", Journal of Aesthetics and Art Criticism, 53, S. 71-75.

Raspa, Venanzio (2006), "Fictional and Aesthetic Objects: Meinong's Point of View", in A. Bottani \& R. Davies [eds.] (2006), Modes of Existence. Papers in Ontology and Philosophical Logic, Frankfurt et al., Ontos Verlag, S. 47-80. 
Rorty, Richard (2003), „Der Roman als Mittel zur Erlösung aus der Selbstbezogenheit", in C. Menke \& J. Küper [Hrsg.] (2003), Dimensionen ästhetischer Erfahrung, Frankfurt, Suhrkamp.

Ryle, Gilbert (1963), The Concept of Mind, Middlesex, Peregrine.

Saxinger, Robert (1902a), "Dispositionspsychologisches über Gefühlskomplexionen", Zeitschrift für Psychologie und Physiologie der Sinnesorgane, 30, S. 391-421.

Saxinger, Robert (1902b), "Über den Einfluss der Gefühle auf die Vorstellungsbewegung", Zeitschrift für Psychologie und Physiologie der Sinnesorgane, 27, S. 18-33.

Saxinger, Robert (1904), „Über die Natur der Phantasiegefühle und Phantasiebegehrungen“, in A. Meinong [Hrsg.] (1904), Untersuchungen zur Gegenstandstheorie und Psychologie, Leipzig, Barth, S. 579-606.

Saxinger, Robert (1906), "Beiträge zur Lehre von der emotionalen Phantasie", Zeitschrift für Psychologie und Physiologie der Sinnesorgane, 40, S. 145-160.

Saxinger, Robert (1908), "Gefühlssuggestion und Phantasiegefühl”, Zeitschrift für Psychologie und Physiologie der Sinnesorgane, 46, S. 401428.

Scheler, Max (1976), "Idealismus-Realismus", in Gesammelte Werke, Bd. IX, Bern und München, Francke, S. 183-242.

Schwarz, Ernst (1906a), "Über Phantasiegefühle", Archiv für die systematische Philosophie, 11, S. 481-496.

Schwarz, Ernst (1906b), “ Über Phantasiegefühle”, Archiv für systematische Philosophie, 12, S. 84-103.

Smith, Barry (1994), Austrian Philosophy. The Legacy of Franz Brentano, Chicago, Open Court.

Solomon, Robert C. (1993), The Passions: Emotions and the Meaning of Life, Indianapolis, Hacket.

Stocker, Michael (1987), "Emotional Thoughts", American Philosophical Quarterly, 24 (1), S. 59-69. 
Stumpf, Carl (1928), Gefühl und Gefühlsempfindung, Leipzig, Barth.

Tappolet, Christine (2000), Émotions et Valeurs, Paris, Presses Universitaires de France.

Tatarkiewicz, Władysław (2003), Geschichte der sechs Begriffe: Kunst, Schönheit, Form, Kreativität, Mimesis, ästhetisches Erlebnis, Frankfurt, Suhrkamp.

Taylor, Gabriele (1985), Pride, Shame and Guilt. Emotions of Selfassessment, Oxford, Clarendon Press.

Utitz, Emil (1911), Die Funktionsfreuden im ästhetischen Verhalten, Halle, Niemeyer.

Vendrell Ferran, Íngrid (2009), „Meinongs Philosophie der Gefühle und ihre Einfluss auf die Grazer Schule“, in Meinong Studies / Meinong Studien, vol. 3, Frankfurt et al., Ontos Verlag, S. 199-239.

Voigtländer, Eise (1910), Über die Typen des Selbstgefühls, Leipzig, R. Voigtländer.

Walton, Kendall (1978), „Fearing Fictions“, The Journal of Philosophy, 75 (1), S. 5-27.

Walton, Kendall (1990), Mimesis as Make-Believe. On the Foundations of the Representational Arts, Harvard, Harvard University Press.

Weston, Michael (1975), "How can we be moved by the fate of Anna Karenina?", Proceedings of the Aristotelian Society, Supplementary Volume XLIX, S. 81-94.

Witasek, Stephan (1904), Grundzüge der Allgemeinen Aesthetik, Leipzig, Barth.

Wollheim, Richard (1984), The Thread of Life, Cambridge (Mass.), Harvard University Press.

Yanal, Robert J. (1999), Paradoxes of Emotion and Fiction, Pennsylvania, The Pennsylvania State University Press. 